\title{
EFEITO DA CHUVA SOBRE A EFICÁCIA DE DIFERENTES FORMULAÇÕES DE GLYPHOSATE NO CONTROLE DE Bidens pilosa
}

\author{
Ricardo Camara Werlang ${ }^{1}$, Antônio Alberto da Silva ${ }^{2}$,Lino Roberto Ferreira ${ }^{3,}$ Glauco \\ Vieira Miranda 4
}

\author{
${ }^{1}$ Engenheiro - Agrônomo, Mestrando, UFV, Av. P.H. Rolfs s/n, Dep. de Fitotecnia; CEP: 36571-000, \\ Viçosa-MG. E-mail: rwerlang @ hotmail.com, Tel. (31) 3891-0319 \\ ${ }^{2}$ Prof. do Dep. de Fitotecnia da UFV, Av. P.H. Rolfs s/n, Depto. de Fitotecnia; CEP: 36571-000, Viçosa- \\ MG. E-mail: aasilva@mail.ufv.br. \\ ${ }^{3}$ Prof. do Dep. de Fitotecnia da UFV, Av. P.H. Rolfs s/n, Depto. de Fitotecnia; CEP: 36571-000, Viçosa- \\ MG. E-mail: 1roberto@ mail.ufv.br. \\ ${ }^{4}$ Prof. do Dep. de Fitotecnia da UFV, Av. P.H. Rolfs s/n, Depto. de Fitotecnia; CEP: 36571-000, Viçosa- \\ MG.
}

\section{RESUMO}

Foi objetivo deste trabalho avaliar os efeitos de doses crescentes de diferentes sais de glyphosate - amônio (Roundup WG), isopropilamina (Roundup Transorb) e potássico (Zapp Qi) - sobre as plantas de Bidens pilosa em condições controladas, quando submetidas à chuva de $20 \mathrm{~mm}$ durante 30 minutos, nos intervalos de 1, 2, 4, 6, 12 e 24 horas após aplicação desses produtos. A eficiência de controle de B. pilosa pelos sais de glyphosate foi afetada quando ocorreu chuva após aplicação. A redução no controle foi maior à medida que se diminuía o intervalo sem chuva após a aplicação. O controle visual de $B$. pilosa foi semelhante dos 7 aos 28 DAA e não diferiu na redução de matéria seca acumulada, em relação à testemunha sem herbicida, para todos os sais de glyphosate. As doses do herbicida se diferenciaram no controle da espécie, sendo o controle mais eficiente nas maiores doses. $\mathrm{O}$ sal isopropilamina demonstrou melhor controle da espécie nos menores intervalos sem chuva após a aplicação (1 e 2 horas), sendo superior aos demais sais. No entanto, nos intervalos sem chuva maiores do que duas horas o sal amônio foi superior no controle visual da espécie. O sal potássico proporcionou controle inferior ao dos demais nos períodos sem chuva inferiores a 12 horas, sendo superior ao sal isopropilamina nos intervalos sem chuva maiores que 12 horas. O sal amônio, mesmo apresentando excelente controle da espécie nas avaliações visuais, proporcionou menor redução na matéria seca do que os demais sais. $\mathrm{O}$ sal isopropilamina proporcionou maior redução da matéria seca de B. pilosa do que os demais tratamentos.

Palavras-chave:sal potássico, sal isopropilamina, sal amônio, glyphosate SA, glyphosate WG.

\section{RAINFALL EFFECTS ON THE EFFECTIVENESS OF THE DIFFERENT GLYPHOSATE FORMULATIONS IN CONTROLLING Bidens pilosa}

\begin{abstract}
This study aimed at evaluating the effects from increasing doses of different glyphosate salts - ammonium (Roundup WG), isopropylamine (Roundup Transorb) and potassic salt (Zapp Qi) - on Bidens pilosa plants under controlled conditions, when they were subjected to a 20-mm rainfall over 30 minutes, at intervals of 1, 2, 4, 6, 12 and 24 hours after application of these products. The efficiency of the glyphosate salts in controlling B. pilosa were affected when the rainfall occurred after their application. The reduction in control was higher as the interval without rainfall after these product applications was diminished. The visual control of B. pilosa was similar from 7 to 28 days after application (DAA) for all these
\end{abstract}


products, and it did not differ as to reduction of the accumulated dry matter in relation to the control without herbicide. The herbicide doses differed in controlling this species, and the most efficient control was obtained with the highest doses. The isopropylamine salt showed a better control upon this species at the shorter intervals without rainfall after its application. (1 and 2 hours), showing to be superior to the other salts. In those intervals without rainfall and longer than two hours, however, the ammonium salt was superior as to the visual control of this species. The potassic salt provided a lower control than the other salts over the periods without rainfall shorter than 12 hours, but showed to be superior to the isopropylamine salt at those intervals without rainfall and longer than 12 hours. Although presenting an excellent control upon the species in visual evaluations, the ammonium salt provided a lower reduction in dry matter than the other salts. The isopropylamine salt provided a higher reduction in dry matter of $B$. pilosa than the other treatments.

Key-Words:potassic salt, isopropylamine salt, ammonium salt, glyphosate SA, glyphosate WG.

\section{INTRODUÇÃO}

O controle eficiente das plantas daninhas depende do herbicida, da dose correta e de uma aplicação adequada. Assim, no campo, a ocorrência de condições climáticas inadequadas no momento da aplicação ou após esta pode ocasionar redução da eficácia dos produtos no controle de plantas daninhas, podendo até inviabilizar essa prática.

O glyphosate pertence ao grupo dos inibidores da síntese de aminoácidos e contém o $\mathrm{N}$-(phosphonomethyl) glycina como ingrediente ativo. É um herbicida sistêmico, nãoseletivo, altamente solúvel em água e seu mecanismo de ação baseia-se na interrupção da rota do ácido chiquímico, responsável pela produção dos aminoácidos aromáticos fenilalanina, tirosina e triptofano, que são essenciais para a síntese de proteínas e divisão celular nas regiões meristemáticas da planta (Hess, 1994). Atualmente são vários os sais utilizados na formulação de glyphosate: potássico (Zapp Qi), isopropilamina (Roundup Transorb e Roundup) e amônio (Roundup WG e Roundup Multiação). Na maioria das plantas, o glyphosate é rapidamente translocado das folhas tratadas para os drenos metabólicos, especialmente tecidos meristemáticos e de armazenagem (Bromilow et al., 1990), sendo, por isso, excelente herbicida para o controle de plantas daninhas perenes.

Após atingir a superfície foliar, o herbicida está sujeito a vários destinos: pode escorrer, ser lavado, secar como uma substância amorfa, cristalizar-se após a evaporação do solvente ou, ainda, penetrar na cutícula e permanecer retido nela, não sendo translocado. A consequiência imediata é a redução na eficiência de ação do herbicida e, portanto, o controle inadequado das plantas daninhas (Devine \& Born, 1991).

Assim, a ocorrência de chuvas após as aplicações interfere na eficácia dos herbicidas aplicados em pós-emergência, reduzindo o controle das plantas daninhas (Bryson, 1988; Wicks et al., 1993; Pires, 1998). A penetração e absorção, retenção superficial e lavagem do herbicida dependem do tipo de herbicida, dos carregadores, diluentes, adjuvantes, da volatilização, da espécie e do estádio de desenvolvimento da planta daninha, bem como dos fatores climáticos. A interação de todos esses fatores proporciona maior ou menor controle da espécie. O conhecimento detalhado dessas interações possibilita melhor aproveitamento do potencial de cada herbicida.

As diferentes formulações do herbicida podem alterar a sua absorção e translocação do ingrediente ativo na planta, influenciando a eficiência do controle das espécies daninhas (Silva et al., 2000). Pesquisas mostram que o glyphosate requer período mínimo de quatro horas livre de chuva após a sua aplicação para controlar eficientemente as plantas daninhas (Bryson, 1988). Segundo Pires (1998), nas formulações tradicionais, tanto para sulfosate como para glyphosate esse período sem chuva após aplicação deve ser de, no mínimo, seis horas para Brachiaria brizantha, e as plantas devem estar em plena atividade metabólica. 
Quanto ao controle de Digitaria horizontalis, Jakelaitis et al. (2001) observaram que as formulações de glyphosate Roundup Transorb, Zapp Qi e sulfosate (Zapp) apresentaram controle da espécie quando o intervalo sem chuva foi de 4-6 horas. Todavia, as formulações de glyphosate Roundup CS e Roundup WG foram mais afetadas pela chuva após as aplicações, quando comparados ao Zapp Qi e Roundup Transorb.

Considerando que esse tempo sem chuva necessário para que o herbicida tenha máxima eficácia pode variar para cada formulação, este trabalho teve como objetivo avaliar a eficácia de diferentes sais de glyphosate - amônio (Roundup WG), isopropilamina (Roundup Transorb) e potássico (Zapp Qi), submetidos à chuva de $20 \mathrm{~mm}$ durante 30 minutos, nos intervalos de 1, 2, 4, 6, 12 e 24 horas após aplicação desses produtos - sobre plantas de Bidens pilosa.

\section{MATERIAL E MÉTODOS}

O experimento foi realizado em casa de vegetação, no período de maio a agosto de 2001, e teve como unidade experimental quatro plantas de Bidens pilosa cultivadas em um vaso com 3,0 L de substrato (solo + fertilizantes). A irrigação dos vasos ocorreu diariamente, procurando manter o solo próximo a $80 \%$ da capacidade de campo, utilizando-se um medidor de potencial de água no solo (Floral Sensor). Foram ainda realizadas adubações de cobertura com adubo Ouro Verde (15-15-20 NPK + Ca, S, Mg, Zn, B, Fe e Mn) em intervalos de sete dias.

Decorridos 35 dias do transplantio, quando as plantas de B. pilosa apresentavam 55 $\mathrm{cm}$ de altura e algumas (cerca de $25 \%$ ) possuíam flores, foram aplicados os tratamentos (Tabela 1). Para isso, utilizou-se um pulverizador costal pressurizado com $\mathrm{CO}_{2}$, mantendo-se a pressão constante em $2,5 \mathrm{kgf} \mathrm{cm}^{-2}$, aplicando-se $200 \mathrm{~L} \mathrm{ha}^{-1}$ de calda, com uma barra possuindo dois bicos XR-110.03. As aplicações foram feitas em intervalos de 24, 12, 6, 4, 2 e 1 hora antecedendo a simulação da chuva, em condições de umidade relativa de $80 \pm 10 \%$ e temperatura de $24 \pm 1^{\circ} \mathrm{C}$. Em seguida, respeitando os períodos sem chuva após a aplicação dos tratamentos, os vasos foram levados para o simulador de chuva, onde receberam lâmina de $20 \mathrm{~mm}$ de água durante 30 minutos. A água do simulador apresentava-se com pH 6,07 e temperatura de $21^{\circ} \mathrm{C}$.

Os tratamentos foram dispostos em delineamento experimental inteiramente casualizado, no esquema fatorial $(3 \times 4 \times 6)+1$, sendo constituído pelos três herbicidas - sal potássico (Zapp Qi), sal isopropilamina (Roundup Transorb) e sal amônio (Roundup WG) -, pelas quatro doses (180, 360, 720 e $1.440 \mathrm{~g} \mathrm{ha}^{-1}$ de eq.ac.) de glyphosate, por seis intervalos sem chuva após a aplicação dos herbicidas $(1,2,4,6,12$ e 24 horas) e pela testemunha sem herbicida, não recebendo chuva, com três repetições.

A eficiência de controle de B. pilosa foi avaliada aos 7, 14, 21 e 28 dias após a aplicação (DAA), com base nos sintomas de fitotoxicidade, utilizando a escala de Frans (1972), em que $0 \%$ correspondeu à ausência de sintomas visíveis e 100\%, à morte das plantas. Aos 28 DAA, as plantas foram cortadas e o material vegetal foi secado em estufa de circulação forçada a $70{ }^{\circ} \mathrm{C}$, até adquirir peso constante. Os resultados de massa seca foram expressos em porcentagem de redução de crescimento em relação à testemunha sem herbicida.

As temperaturas máxima e mínima e a umidade relativa do ar no interior da casa de vegetação durante o período do experimento estão apresentadas na Figura 1.

Para interpretação dos resultados de fitotoxicidade, procedeu-se à análise de variância, sendo as médias comparadas pelo teste de Tukey a 5\% de probabilidade. As interações significativas foram estudadas por análise de regressão e superfície de resposta, determinando a melhor função que explicasse os resultados obtidos pelo software SAEG (Ribeiro Jr., 2001). As funções foram trabalhadas para visualização gráfica pelo software 
STATISTICA (1999). Para melhor visualização dos resultados obtidos, o controle de $B$. pilosa foi dividido em cinco faixas, sendo elas: 90 a 100\% - controle excelente ou total da espécie; 80 a $90 \%$ - controle bom; 70 a 80\% - controle moderado; 50 a $70 \%$ - controle deficiente ou inexpressivo; e < que 50\% - ausência de controle.

\section{RESULTADOS E DISCUSSÃO}

Após a realização das análises de variância (Tabela 2), as interações significativas foram estudadas por superfície de resposta, determinando-se a melhor função que explicasse os resultados analisados.

Os resultados da eficiência de controle de B. pilosa pelos diferentes sais de glyphosate - potássico (Zapp Qi), isopropilamina (Roundup Transorb) e amônio (Roundup WG) - em função das doses e do intervalo de tempo sem chuva após aplicação podem ser observados na Figura 2a,b,c. A superfície de controle visual aos 28 DAA (>90\%), ou seja, a combinação de doses e intervalos sem chuva, foi maior para o sal amônio do que para os outros sais. Entretanto, nos menores intervalos sem chuva após a aplicação (uma e duas horas), o sal isopropilamina (Roundup Transorb) foi superior, apresentando controle maior do que $90 \%$ na dose de $1.440 \mathrm{~g} \mathrm{ha}^{-1}$ de eq.ac. para uma hora livre de chuva (Figura $2 \mathrm{~b}$ ), ao passo que nos demais sais o controle ficou entre 80 e $89 \%$, nas mesmas condições (Figura 2a,c).

A ocorrência de chuva após a aplicação de glyphosate proporcionou redução no controle de $B$. pilosa pelo herbicida. Essa redução na eficiência do glyphosate aumentou com a diminuição do intervalo de tempo entre a aplicação do herbicida e a ocorrência da chuva (Figura 2a,b,c). Resultados semelhantes foram obtidos por Bryson (1988), Pires (1998) e Jakelaitis et al. (2001).

Os efeitos dos diferentes sais de glyphosate podem ser observados na Tabela 3. Todos os sais de glyphosate apresentaram controle de B. pilosa semelhante dos 7 aos 21 DAA. Aos 28 DAA, o sal isopropilamina (Roundup Transorb) proporcionou controle semelhante ao do sal amônio (Roundup WG) e superior ao do sal potássico (Zapp Qi). No entanto, agronomicamente essa diferença não é considerada, pois o controle ficou entre 76 e $79 \%$ (respectivamente para os sais potássico e isopropilamina), diferença de apenas $3 \%$ de controle, sendo de difícil visualização em campo.

Todos os sais de glyphosate proporcionaram redução no acúmulo de matéria seca da parte aérea de $B$. pilosa aos 28 DAA (Tabela 3), ocorrendo menor acúmulo nos tratamentos com o sal isopropilamina, não diferindo do sal potássico. No entanto, agronomicamente, de modo semelhante ao observado na avaliação do controle visual, esses sais não diferiram entre si, uma vez que a diferença foi pequena e estes não diferiram na redução de matéria seca acumulada, em relação à testemunha sem herbicida.

A superfície de redução da matéria seca acumulada de $B$. pilosa, em relação à testemunha sem herbicida e sem chuva, aos 28 DAA, para os sais de glyphosate nas doses e nos intervalos sem chuva após aplicação avaliados, encontra-se na Figura 3a,b,c.

As doses de glyphosate se diferenciaram no controle de $B$. pilosa, observando-se melhora na eficácia com o acréscimo das doses. Esse fato foi confirmado para o acúmulo de matéria seca (maior redução nas maiores doses) (Figuras 2 e 3). Nos menores intervalos sem chuva após a aplicação o efeito das doses foi mais pronunciado no controle da espécie. Isso fica evidente quando se observa que, no intervalo de uma hora sem chuva após aplicação, o sal isopropilamina (Figura 2b) demonstrou superfície de 50 a $69 \%$ de controle na menor dose até controle maior do que $90 \%$ na maior dose, diferindo dos resultados obtidos no controle de Brachiaria decumbens (dados não publicados), em que o efeito das doses no controle da espécie foi mais evidente nos intervalos superiores a seis horas sem chuva após aplicação.

A dose do herbicida teve influência na maior quantidade absorvida, translocada e que alcança o local de ação. Portanto, doses maiores exigem menor intervalo sem chuva, para controle eficiente. Esses resultados estão de acordo com os observados por Wicks et al. 
(1993), os quais verificaram redução do controle de Echinochloa colonum na dose de $225 \mathrm{~g}$ ha $^{-1}$ de glyphosate com a ocorrência de chuva de $3 \mathrm{~mm}$ após aplicação. Nessas condições, foi necessário intervalo de 45 horas sem chuva para obter controle de 73\%. No entanto, na dose de $900 \mathrm{~g} \mathrm{ha}^{-1}$ de glyphosate, controle de $93 \%$ foi alcançado com o tempo de seis horas sem chuva após aplicação.

Todos os sais de glyphosate foram afetados pela ocorrência de chuva após aplicação (20 mm em 30 minutos), sendo esse efeito mais pronunciado nas menores doses, como observado na dose de $360 \mathrm{~g} \mathrm{ha}^{-1}$ de eq.ac. do sal amônio (Figura 2c), que demonstrou superfície de 50 a $69 \%$ de controle com intervalo livre de chuva após aplicação de uma hora até controle da espécie maior do que $90 \%$ no intervalo de 24 horas sem chuva. Esses resultados diferiram daqueles obtidos no controle de $B$. decumbens (dados não publicados), em que o efeito dos intervalos livres de chuva foi mais pronunciado no controle da espécie nas maiores doses de glyphosate (1.440 e $2.160 \mathrm{~g} \mathrm{ha}^{-1}$ de eq.ac.), independentemente do sal.

Para o Zapp Qi (sal potássico) na dose de $720 \mathrm{~g} \mathrm{ha}^{-1}$ de eq.ac. (Figura 2a), foi necessário intervalo de pelo menos 12 horas sem chuva após aplicação para se obter controle da espécie maior que $90 \%$, diferindo dos resultados obtidos por Jakelaitis et al. (2001) para controle de Digitaria horizontalis, em que o intervalo de seis horas sem chuva após aplicação não diferiu do tratamento sem chuva.

A eficiência do controle das plantas daninhas pelos herbicidas é dependente de sua ação na planta. É necessário, no entanto, que a molécula seja absorvida e translocada até o local de ação, em quantidade adequada, onde desempenhará sua função. Até alcançar o local de ação, a molécula deve passar por várias barreiras, e o glyphosate sozinho não passa facilmente por todas elas na planta. Assim, a formulação de glyphosate é de extrema importância na sua eficiência, uma vez que são misturadas ao princípio ativo substâncias que melhoram as condições de permanência, absorção, solubilidade e translocação na planta (Laerke \& Streibig, 1995). As formulações do glyphosate são compostas por diferentes sais e surfatantes, e estes possuem comportamento diferenciado quanto à sua ação e interação (Laerke \& Streibig, 1995), justificando, assim, a variação na resposta no controle de B. pilosa pelas diferentes formulações desse herbicida.

A taxa de absorção das diferentes formulações de glyphosate é superior à medida que a gota aplicada está secando (Stevens et al., 1991), devido ao incremento da concentração do herbicida enquanto a gota perde água. Segundo Liu et al. (1996), a formação de maior gradiente de concentração entre a gota e a folha e a sua maior permanência nesta são os principais fatores que aumentam a absorção. A formulação do glyphosate influencia, ainda, o tempo de permanência da gota, em condição adequada de absorção sobre a folha (Nalewata et al., 1996), sem que ocorra a formação de cristal ou forma amorfa, afetando, assim, a velocidade de absorção e a quantidade do herbicida absorvido, o que é refletido na maior eficácia de controle das plantas daninhas e, conseqüentemente, em menores perdas pela ocorrência de chuva após aplicação. Isso também foi observado no sal isopropilamina, que foi menos afetado do que os demais sais nos intervalos de uma e duas horas livres de chuva após a aplicação, tanto no controle visual como na redução da matéria seca acumulada aos 28 DAA (Figuras $2 b$ e $3 b$ ).

O glyphosate na dose de $720 \mathrm{~g} \mathrm{ha}^{-1}$ de eq.ac., com os sais de isopropilamina e amônio (Figura 2b,c) proporcionou controle de B. pilosa de 80 a $89 \%$ quando a chuva ocorreu em intervalos de quatro e seis horas após as aplicações e controle maior do que $90 \%$ no intervalo de 12 horas sem chuva após aplicação, aos 28 DAA. Nesta dose, foi necessário intervalo de pelo menos 7, 12 e 12 horas sem chuva após aplicação para se observar controle visual da espécie maior que $90 \%$, respectivamente para os sais amônio, isopropilamina e potássico (Figura 2a,b,c).

Comparando os efeitos dos sais em cada intervalo sem chuva após aplicação e em cada dose, na avaliação de controle visual, verificou-se que o Zapp Qi (sal potássico) foi 
menos eficiente que os demais herbicidas nos menores intervalos sem chuva, exigindo doses maiores. No entanto, foi mais eficiente que o sal isopropilamina nos maiores intervalos sem chuva (> 12 horas), diferindo do observado por Jakelaitis et al. (2001), em cujo estudo o sal potássico (Zapp Qi) e o sal isopropilamina (Roundup Transorb) foram menos afetados do que o sal amônio (Roundup WG) pela ocorrência de chuva no controle de Digitaria horizontalis. Esses resultados diferiram daqueles observados no controle de Brachiaria decumbens, em que os sais potássico e isopropilamina sofreram menor efeito da ocorrência de chuva no controle da espécie do que o sal amônio (dados não publicados).

O Roundup Transorb (sal isopropilamina) demonstrou melhor controle da espécie nos menores intervalos sem chuva após a aplicação (uma e duas horas) (Figura 2b), sendo superior aos demais sais. Entretanto, nos maiores intervalos sem chuva (> 2 horas), o Roundup WG (sal amônio) foi superior no controle visual da espécie (Figura 2c). O sal potássico (Zapp Qi) proporcionou controle inferior ao dos demais nos períodos sem chuva inferiores a 12 horas (Figura 2a), sendo superior ao sal isopropilamina nos intervalos sem chuva maiores que 12 horas.

Para demonstrar controle da espécie maior que $90 \%$ aos 28 DAA, o sal amônio (Roundup WG) exigiu intervalo de pelo menos duas horas sem chuva após aplicação na dose de $1.440 \mathrm{~g} \mathrm{ha}^{-1}$ de eq.ac. de glyphosate; todavia, na dose de $720 \mathrm{~g} \mathrm{ha}^{-1}$ de eq.ac. de glyphosate foi necessário período de pelo menos sete horas para se obter o mesmo controle. $\mathrm{O}$ sal isopropilamina (Roundup Transorb) necessitou de intervalo sem chuva de 1 e 12 horas sem chuva (respectivamente para as doses de 1.440 e $720 \mathrm{~g} \mathrm{ha}^{-1}$ de eq.ac. de glyphosate) para demonstrar controle da espécie maior que $90 \%$ aos 28 DAA. No entanto, o sal potássico (Zapp Qi) apresentou o mesmo nível de controle na maior dose $\left(1.440 \mathrm{~g} \mathrm{ha}^{-1}\right.$ de eq.ac. de glyphosate) a partir de cinco horas sem chuva, exigindo intervalos superiores a 12 horas na dose de $720 \mathrm{~g} \mathrm{ha}^{-1}$ de eq.ac. de glyphosate.

As diferenças observadas no efeito das doses e da ocorrência de chuva após aplicação e suas interações, no controle de B. pilosa, mesmo trabalhando com quantidades iguais de equivalente ácido do herbicida nas formulações de glyphosate, possivelmente se devem às diferentes taxas de absorção dos sais. Segundo Nalewaja et al. (1996), a absorção relativa à quantidade aplicada foi de 59\% para o sal isopropilamina, $44 \%$ para o sal amônio, $21 \%$ para o sal sódico e $1 \%$ para o sal cálcico. Contudo, a taxa de absorção dos diferentes sais é, também, influenciada pela interação destes com os surfatantes presentes nas formulações. Nalewaja et al. (1996), estudando o efeito de vários sais de glyphosate na redução da matéria seca de trigo, observaram que todas as formulações de glyphosate foram menos eficientes quando aplicadas com X-77 do que com MON 0818 ou Tween-20 a 1\% na solução, sendo o sal isopropilamina o mais ativo, independentemente do surfatante.

O sal amônio (Roundup WG), mesmo apresentando excelente controle da espécie nas avaliações visuais, demonstrou menor redução na matéria seca da parte aérea, quando comparado com os sais isopropilamina (Roundup Transorb) e potássico (Zapp Qi) (Figura 3a,b,c). O Roundup Transorb (sal isopropilamina) apresentou maior redução da matéria seca de Bidens pilosa do que os demais tratamentos, demonstrando, assim, que o maior controle visual proporcionado pelo sal amônio não se refletiu na redução da matéria seca, possivelmente devido à maior ocorrência de necrose nos tecidos vegetais dos tratamentos com este sal, que não evoluiu para a morte da planta. A morte das células no local de contato do herbicida com estas pode proporcionar refluxo e perda do glyphosate absorvido pela planta, ocasionando menor translocação do herbicida e, consequientemente, menor controle da espécie (Morin et al., 1998). Segundo Gaskin \& Zabkiewicz (1992), a perda da integridade da membrana celular reduz a translocação, aumenta a concentração do ingrediente ativo em determinados pontos e ocasiona a morte localizada de células (intoxicação de contato), proporcionando a redução na eficácia do glyphosate.

A fim de proporcionar redução da matéria seca superior a $80 \%$ o sal potássico 
demandou intervalo de pelo menos 13 horas sem chuva após a aplicação, na maior dose. O sal isopropilamina necessitou de intervalo de seis horas sem chuva para proporcionar o mesmo efeito na redução do acúmulo de matéria seca, também na maior dose. No entanto, o sal amônio proporcionou redução da matéria seca superior a $80 \%$ somente a partir do intervalo de 23 horas sem chuva, na mesma dose dos demais sais. A redução total da matéria seca só foi ocasionada por Roundup Transorb (sal isopropilamina) na maior dose com intervalo sem chuva superior a 21 horas.

O glyphosate é facilmente influenciado pela ocorrência de chuva após aplicação devido à sua absorção relativamente lenta, conforme observado por Bryson (1988), estudando o efeito de intervalos sem chuva $(12,7 \mathrm{~mm}$ em 10 minutos) no controle de plantas novas de Sorghum halepense, por vários herbicidas. O autor observou que os herbicidas ácido fosfonados (glyphosate e SC-0224) precisaram de períodos superiores aos exigidos pelos herbicidas seletivos estudados. De acordo com Bariuan et al. (1999), a absorção de $C^{14}$ glyphosate sal isopropilamina aumentou de $2,84 \%$, uma hora após aplicação, para 4,18; 6,42; 8,35; e 21,39\%, respectivamente 6, 24, 72 e 168 horas após aplicação de Roundup Ultra (560 $\mathrm{g} \mathrm{ha}^{-1}$ do produto comercial) em Cyperus rotundus.

As diferentes respostas das formulações estudadas na redução da matéria seca acumulada, quanto à ocorrência de chuva após aplicação e sua interação com as doses, possivelmente se devem às diferentes taxas de absorção de glyphosate para cada formulação. Feng et al. (2000), estudando a absorção de $\mathrm{C}^{14}$-glyphosate por Abutilon theophasti, observaram, no intervalo de 120 horas após a aplicação, que a absorção de Roundup Ultra, Roundup Original e Sulfosate 5 foi, respectivamente, de 36,2, 31,8 e 22,7\%; entretanto, 24 horas após a aplicação, apresentaram 27,8, 16,0 e 12,72\% de absorção, respectivamente para Roundup Ultra, Sulfosate 5 e Roundup Original, aplicados na dose de $200 \mathrm{~g} \mathrm{ha}^{-1}$ de eq.ac. Isso demonstra que a taxa de absorção varia para as diferentes formulações e também é afetada no decorrer do tempo, podendo determinada formulação possuir melhor absorção em períodos iniciais após aplicação, diminuindo posteriormente. Os mesmos autores, comparando Sulfosate 5 e Roundup Original, observaram que 24 horas após aplicação o primeiro apresentou maior absorção; contudo, 120 horas após, o Roundup Original apresentou maior taxa de absorção de glyphosate.

O herbicida ainda não absorvido está vulnerável à ação da chuva, que pode lavá-lo da superfície foliar, ocasionando redução do controle das plantas daninhas e conseqüentemente perdas econômicas, além do risco potencial de contaminação do ambiente. O glyphosate apresenta, em geral, baixo potencial de contaminação do solo, uma vez que é fortemente adsorvido às suas partículas constituintes e rapidamente degradado pelos microrganismos do solo (Sprankle et al., 1975; Rodrigues \&Almeida, 1998), embora o resíduo do glyphosate, em solos arenosos e com baixa atividade microbiana, possa causar injúrias em algumas culturas (Cornish, 1992; Piccolo et al., 1994).

As diferentes formulações de glyphosate afetam a quantidade do herbicida lavado da superfície foliar, como observado por Feng et al. (2000). As diferenças foram marcantes com a chuva ocorrendo 24 horas após a aplicação, em que a porcentagem do Roundup Ultra (sal isopropilamina) recuperado na água da chuva foi de 71,8\%, comparado com 83,8 e 86,7\%, respectivamente para Sulfosate 5 (sal trimetilsulfônico de sulfosate) e Roundup Original (sal isopropilamina). Consequientemente, o Roundup Ultra foi mais eficiente do que os demais na absorção de glyphosate por Abutilon theophrasti, em particular nos menores intervalos (4 e 24 horas após aplicação).

Portanto, a velocidade de absorção do glyphosate é um componente importante no desempenho deste quanto à sua ação herbicida, principalmente na ocorrência de chuva após a aplicação. As diferenças nas eficiências de controle dos sais estudados possivelmente são relacionadas com suas diferentes taxas de absorção e reação na folha, como a ocorrência de necrose no local de absorção, restringindo, assim, a translocação e ação na planta. $O$ 
conhecimento detalhado das diferenças nas taxas de absorção e translocação dos sais de glyphosate e suas formulações é necessário para minimizar as perdas e reduzir o potencial de contaminação do ambiente.

Todos os sais de glyphosate estudados foram afetados, quanto ao controle de Bidens pilosa, pela ocorrência de chuva após aplicação $(20 \mathrm{~mm}$ em 30 minutos).A redução no controle foi maior à medida que se diminuía o intervalo sem chuva após a aplicação. Todos os sais de glyphosate estudados proporcionaram controle de B. pilosa semelhante dos 7 aos 28 DAA e não diferiram na redução de matéria seca acumulada, em relação à testemunha sem herbicida. As doses de glyphosate se diferenciaram no controle de B. pilosa, sendo o controle mais eficiente nas maiores doses (180, 360, 720 e $1.440 \mathrm{~g} \mathrm{ha}^{-1}$ de eq.ac.). Nos menores intervalos sem chuva após a aplicação, o efeito das doses no controle da espécie foi mais pronunciado. Da mesma forma, nas menores doses, o efeito dos intervalos sem chuva após a aplicação foi mais evidente no controle de B. Pilosa. A superfície de controle aos 28 DAA (maior que 90\%), ou seja, a combinação de doses e intervalos sem chuva, foi maior para o sal amônio do que para os outros sais. O Roundup Transorb (sal isopropilamina) proporcionou melhor controle da espécie nos menores intervalos sem chuva após a aplicação (uma e duas horas), sendo superior aos demais sais. No entanto, nos maiores intervalos sem chuva (> 2 horas), o Roundup WG (sal amônio) foi superior no controle da espécie. O sal potássico (Zapp Qi) proporcionou controle inferior ao dos demais nos períodos sem chuva inferiores a 12 horas, sendo superior ao sal isopropilamina nos intervalos sem chuva maiores que 12 horas. O sal amônio (Roundup WG), mesmo apresentando excelente controle da espécie nas avaliações visuais, proporcionou menor redução na matéria seca, quando comparado com os sais isopropilamina (Roundup Transorb) e potássico (Zapp Qi). O Roundup Transorb (sal isopropilamina) apresentou maior redução da matéria seca de Bidens pilosa do que os demais tratamentos.

\section{LITERATURA CITADA}

BARIUAN, J. V.; REDDY, K. N.; WILLS, G. D. Glyphosate injury, rainfastness, absorption, and translocation in purple nutsedge (Cyperus rotundus). Weed Technology, vol.13, p.112-119, 1999.

BROMILOW, R. H.; CHAMBERLAIN, K.; EVANS, A. A. Physiocochemical aspects of phloem translocation of herbicide. Weed Sci., Champaign, v.38, p.305-314, 1990.

BRYSON, C. T. Effects of rainfall on foliar herbicides applied to seedling Johnson grass. Weed Tech., Champaign, v.2, p.153-158, 1988.

CORNISH, P. S. Glyphosate residues in a sandy soil affect tomato transplants. Aust. J. Exp. Agric., v.32, n.3. p.395-399, 1992.

DEUBER, R. Ciência das plantas daninhas: fundamentos. Jaboticabal, SP: FUNEP, v.1, 1992. 431p.

DEVINE, M. D.; BORN, W. H. V. Absorption and transport in plants. In: GROVER, R., CESSNA, A. J. Enviromental chemistry of herbicides. Vol. II. CRC Press, Florida. p.119-140. 302p. 1991.

FENG, P. C. C.; SANDBRINK, J. J.; SAMMONS, R. D. Retention, uptake, and translocation of ${ }^{14} \mathrm{C}$-glyphosate from track-spray applications and correlation to rainfastness in velvetleaf (Abutilon theophrasti). Weed Technology, vol.14, p.127-132, 2000. 
FRANS, R. E. Measuring plant responses. In: WILKINSON, R. E. (Ed.). Research methods in weed Science. Southern Weed Science Society, 1972. P. 28-41.

GASKIN, R. E.; ZABKIEWICZ, J. A. Effect of plant age and adjuvant on the foliar penetration and translocation of glyphosate in pampas grass (Cortaderia selloana). In: FOY, C. L. Adjuvants for agrichemicals. Boca Raton: CRC Press, 1992. 735p., p.405410.

HARTZLER, B. Which glyphosate product is best? (2801/2001) (http:www.weeds.iastate.edu/mgmt/qtr01-1/glyphosateformulations.htm.)

HESS, F. D. Mechanism of action of inhibitors of amino acid biosynthesis. In: Herbicide action: an intensive course on the activity, seletivity, behavior, and fate of herbicides in plants and soil. West Lafayette, Indiana: Purdue university, 1994. p.344-365.

HESS. F. D.; FALK, R. H. Herbicide deposition on the leaf surface. Weed Sci., Champaingn, v.38, p. 280-288, 1990.

JAKELAITIS, A.; FERREIRA, L. R.; SILVA, A. A.; MIRANDA, G. V. Controle de Digitaria horizontalis pelos herbicidas glyphosate, sulfosate e glifosate potássico submetidos a diferentes intervalos de chuva após a aplicação. Planta Daninha, v.19, n.2, p.279-285, 2001.

LAERKE, P. E.; STREIBIG, J. C. Foliar absorption of some glyphosate formulations and their efficacy on plants. Pesticide Science, vol.44, p.107-116, 1995.

LIU, S. H.; CAMPBELL, R. A.; STUDESNS. J. A.; WAGNER, R. G. Absorption and translocation of glyphosate in Aspen (Populus tremuloides Michx.) as influenced by droplet size, droplet number, and herbicide concentration. Weed Science, vol.44, p.482488, 1996.

MORIN, F.; VERA, V.; NURIT, F.; TISSUT, M.; MARIGO, G. Glyphosate uptake in Catharanthus roseus cells: Role of a phosphate transporter. Pesticide Biochemistry and Physiology, vol. 58, p. 13-22, 1997.

NALEWAJA, J. D.; DEVILLIERS, B.; MATYSIAK, R. Surfactant and salt affect glyphosate retention and absorption. Weed Research, vol. 36, p.241-247, 1996.

PICCOLO, A.; CELANO, G.; ARIENZO, M.; MIRABELLA, A. Adsorption and desorption of glyphosate in some european soils. J. Environ. Sci. Health,Part B, v.6, p.1105-1115, 1994.

PIRES, N. M. Efeitos do glyphosate e do sulfosate após simulação de chuva em plantas de Brachiaria brizantha submetidas ao estresse hídrico. Viçosa, UFV, Tese DS, 1998. 100 p.

RIBEIRO JR., J. I. Análise estatística no SAEG. Viçosa: UFV, 2001. 301p.

RODRIGUES, B. N.; ALMEIDA, F. S. Guia de herbicidas, Londrina: Edição dos autores, 1998, $648 \mathrm{p}$. 
SILVA, A. A. da; SILVA, J. F. da; FEREIRA, F. A .; FERREIRA, L. R. \& SILVA, J. F. da. ABAEAS. Módulo 3. Controle de plantas daninhas, 2000. 260 p.

SPRANKLE, P.; MEGGITT, W. F.; PENNER, D. Adsorption, action, and translocation of glyphosate. Weed Sci., v.23, n.3, p.235-240, 1975.

STATISTICA program / documentation. StatSoft, In. (1999). Statistica for Windows (Computer program manual).

STEVENS, P. J. G.; GASKIN, R. E.; HONG, S. O.; ZABKIEWICZ, J. A. Contributions of stomatal infiltration and cuticular penetration to enhancements of foliar uptake by surfactants. Pesticide Science, vol.33, p.371-382, 1991.

WICKS, G. A.; FELTON, W. L.; WELSBY, S. M. Effect of rainfall on glyphosate performance on stressed grass weeds following wheat harverst. Plant Protection Quarterly, v.8, n.1, p.2-6. 1993.

WICKS, G. A.; HANSON, G. E. Effect of rainfall on glyphosate plus 2,4-D performance on Echinochloa crus-galli. Weed Sci., Champaign, v.43, p.666-670, 1995. 
Tabela 1 - Tratamentos avaliados no controle de Bidens pilosa em casa de vegetação. Viçosa, 2001

\begin{tabular}{|c|c|c|c|c|c|c|c|c|}
\hline \multirow[t]{2}{*}{ Produto comercial } & \multicolumn{2}{|r|}{ Dose } & \multicolumn{6}{|c|}{ 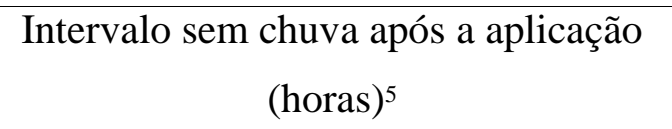 } \\
\hline & $\begin{array}{l}\text { eq. ac. } \\
\text { g ha-1 }\end{array}$ & $\begin{array}{l}\text { p. comercial } \\
\text { L ou kg ha }{ }^{-1}\end{array}$ & & & & & & \\
\hline Zapp Qi ${ }^{1}$ & 180 & 0,36 & 1 & 2 & 4 & 6 & 12 & 24 \\
\hline Zapp Qi ${ }^{1}$ & 360 & 0,72 & 1 & 2 & 4 & 6 & 12 & 24 \\
\hline Zapp Qi ${ }^{1}$ & 720 & 1,44 & 1 & 2 & 4 & 6 & 12 & 24 \\
\hline Zapp Qi ${ }^{1}$ & 1.440 & 2,88 & 1 & 2 & 4 & 6 & 12 & 24 \\
\hline Roundup Transorb ${ }^{2}$ & 180 & 0,38 & 1 & 2 & 4 & 6 & 12 & 24 \\
\hline Roundup Transorb ${ }^{2}$ & 360 & 0,75 & 1 & 2 & 4 & 6 & 12 & 24 \\
\hline Roundup Transorb ${ }^{2}$ & 720 & 1,50 & 1 & 2 & 4 & 6 & 12 & 24 \\
\hline Roundup Transorb ${ }^{2}$ & 1.440 & 3,00 & 1 & 2 & 4 & 6 & 12 & 24 \\
\hline Roundup $\mathrm{WG}^{3}$ & 180 & 0,25 & 1 & 2 & 4 & 6 & 12 & 24 \\
\hline Roundup WG ${ }^{3}$ & 360 & 0,50 & 1 & 2 & 4 & 6 & 12 & 24 \\
\hline Roundup WG ${ }^{3}$ & 720 & 1,00 & 1 & 2 & 4 & 6 & 12 & 24 \\
\hline Roundup WG ${ }^{3}$ & 1.440 & 2,00 & 1 & 2 & 4 & 6 & 12 & 24 \\
\hline Testemunha sem herbicida ${ }^{4}$ & --- & --- & --- & --- & --- & --- & --- & --- \\
\hline
\end{tabular}

${ }^{1 /}$ Formulação comercial (SA) contendo $50 \%$ de glyphosate (sal potássico). ${ }^{2 /}$ Formulação comercial (SA) contendo $48 \%$ de glyphosate (sal isopropilamina). ${ }^{3 /}$ Formulação comercial (WG) contendo $72 \%$ de glyphosate (sal amônio). ${ }^{4 /}$ Testemunha sem herbicida. ${ }^{5 /}$ Aplicação de uma lâmina de $20 \mathrm{~mm}$ de chuva em 30 minutos, no simulador de chuva.

Figura 1 - Dados meteorológicos observados no interior da casa de vegetação - médias por semana - durante o período de realização do experimento, implantado em 1.5.2001 e aplicação dos tratamentos na oitava semana. Viçosa, 2001. 
Tabela 2 - Resumo das análises de variância dos dados referentes às percentagens de controle e matéria seca de Bidens pilosa nas diferentes épocas de avaliação. Viçosa, MG, 2001

\section{Quadrado médio}

Fonte de variação

\begin{tabular}{lcrrrrrr} 
& GL & \multicolumn{1}{c}{ 7 DAA } & 14 DAA & 21 DAA & 28DAA & Matéria seca $^{1}$ & Redução $^{2}$ \\
\hline Herbicidas & 2 & $25,7 \mathrm{~ns}$ & $97,09^{* *}$ & $153,13^{* *}$ & $149,54^{* *}$ & $92,48^{*}$ & $741,32 \mathrm{~ns}$ \\
Doses & 3 & $17041,2^{* *}$ & $8127,5^{* *}$ & $9778,2^{* *}$ & $9672,4^{* *}$ & $3403,79^{* *}$ & $27284,8^{* *}$ \\
Intervalos sem chuva & 5 & $9862,8^{* *}$ & $6215,5^{* *}$ & $3137,4^{* *}$ & $2850,9^{* *}$ & $414,21^{* *}$ & $3320,32^{* *}$ \\
Doses x Herbicidas & 6 & $468,8^{* *}$ & $105,51^{* *}$ & $33,06^{* *}$ & $45,04^{* *}$ & $71,27 \mathrm{~ns}$ & $571,29 \mathrm{~ns}$ \\
Inter. s/ chuva x Herb. & 10 & $286,2^{* *}$ & $97,64^{* *}$ & $148,26^{* *}$ & $164,10^{* *}$ & $24,87 \mathrm{~ns}$ & $199,32 \mathrm{~ns}$ \\
Doses x Inter. s/ chuva & 15 & $924,0^{* *}$ & $151,50^{* *}$ & $109,04^{* *}$ & $136,89^{* *}$ & $60,10^{*}$ & $481,80^{*}$ \\
Herb. x Inter. s/ chuva x Doses & 30 & $204,1^{* *}$ & $46,06^{* *}$ & $30,42^{* *}$ & $31,43^{* *}$ & $39,62 \mathrm{~ns}$ & $317,62 \mathrm{~ns}$ \\
Resíduo & 144 & 39,21 & 8,37 & 6,48 & 6,44 & 33,97 & 272,29 \\
\hline CV (\%) & & 22,9 & 5,8 & 3,6 & 3,3 & 23,1 & 57,7
\end{tabular}

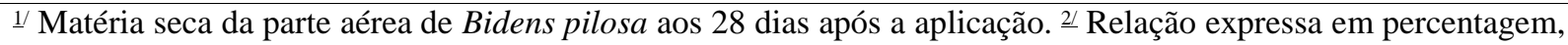
entre a matéria seca dos tratamentos e a testemunha sem herbicida. ** Significativo a $1 \%$ e * $5 \%$ pelo teste de F e n.s. Não-significativo pelo teste de F. Abreviações: Inter. s/ chuva - intervalos sem chuva; Herb. - herbicidas.

Tabela 3 - Resultados médios de controle e matéria seca de Bidens pilosa em função dos diferentes sais de glyphosate, nas avaliações. Viçosa, 2001

Controle visual $(\%)^{5 *} \quad$ Matéria seca acumulada ${ }^{*}$

Herbicidas

\begin{tabular}{|c|c|c|c|c|c|c|}
\hline & 7 DAA & $14 \mathrm{DAA}$ & $21 \mathrm{DAA}$ & $28 \mathrm{DAA}$ & Matéria seca ${ }^{6}$ & Redução $^{7}$ \\
\hline Zapp Qi ${ }^{1}$ & $27 \mathrm{a}$ & $49 \mathrm{a}$ & $70 \mathrm{a}$ & $76 \mathrm{~b}$ & $25,7 \mathrm{bc}$ & $27,4 a$ \\
\hline Roundup Transorb ${ }^{2}$ & $27 \mathrm{a}$ & $51 \mathrm{a}$ & 73 a & 79 a & $23,9 \mathrm{c}$ & 32,2 a \\
\hline Roundup WG ${ }^{3}$ & $28 \mathrm{a}$ & $50 \mathrm{a}$ & $71 \mathrm{a}$ & $78 \mathrm{ab}$ & $26,1 \mathrm{~b}$ & 26,1 a \\
\hline Testemunha ${ }^{4}$ & 0 & 0 & 0 & 0 & 35,3 a & $0,0 \mathrm{~b}$ \\
\hline
\end{tabular}

1/ Formulação comercial (SA) contendo 50\% de glyphosate (sal potássico). ㄹl Formulação comercial (SA) contendo $48 \%$ de glyphosate (sal isopropilamina). 느 Formulação comercial (WG) contendo $72 \%$ de glyphosate (sal amônio). ${ }^{4 /}$ Testemunha sem herbicida. $\underline{5}$ / Média de 72 parcelas, sendo todos os tratamentos de intervalos sem chuva (Aplicação de lâmina de $20 \mathrm{~mm}$ de chuva, em 30 minutos) após a aplicação (1, 2, 4, 6, 12 e 24 horas). 1 l Matéria seca da parte aérea obtida aos 28 dias após a aplicação. ㄱ Relação, expressa em percentagem, entre a matéria seca dos tratamentos e a testemunha sem herbicida. * Médias seguidas pelas mesmas letras na coluna não diferem entre si, pelo teste de Tukey, a 5\% de significância. 
a)

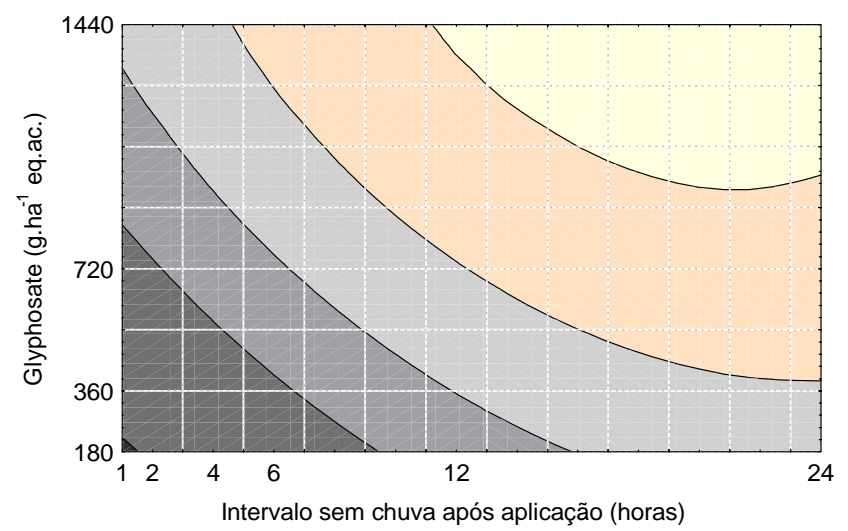

b)

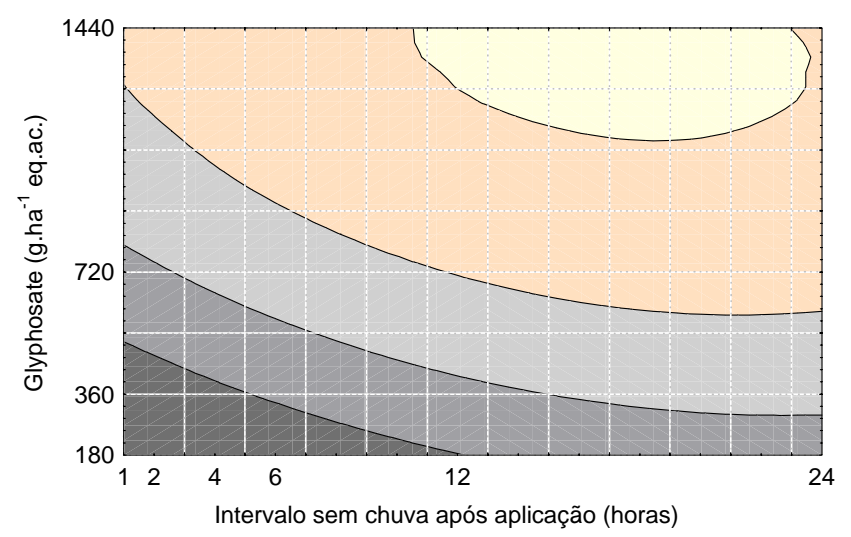

c)

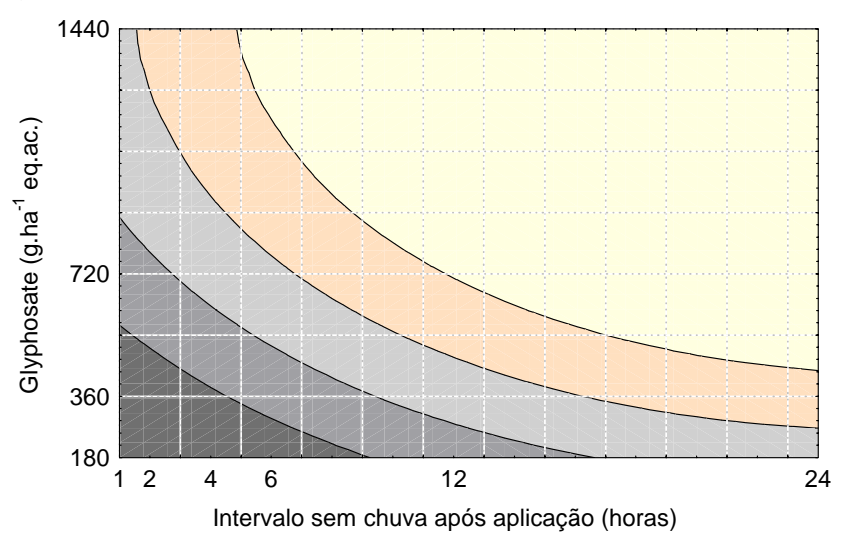

Figura 2 - Porcentagem de controle de Bidens pilosa aos 28 dias após a aplicação (DAA), em função das doses de Zapp Qi (sal potássico) (a), Roundup Transorb (sal isopropilamina) (b), Roundup WG (sal amônio) (c) e dos intervalos sem chuva após aplicação (20 mm em 30 minutos). 
a)

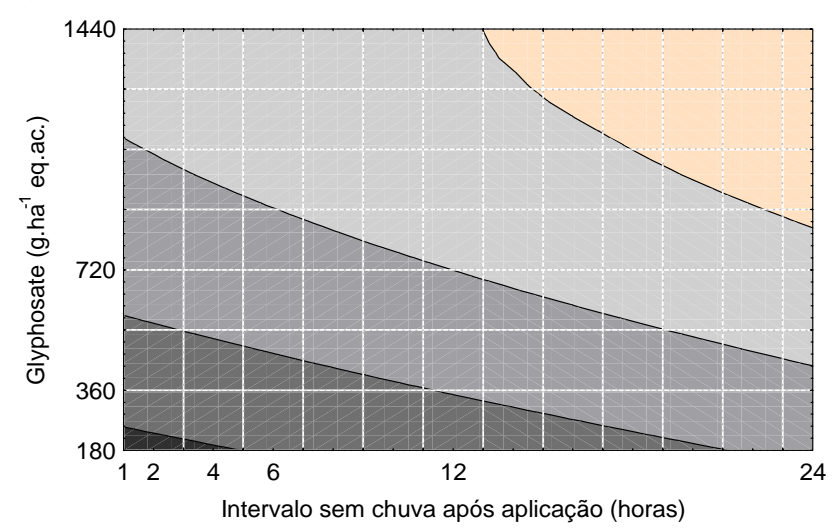

b)

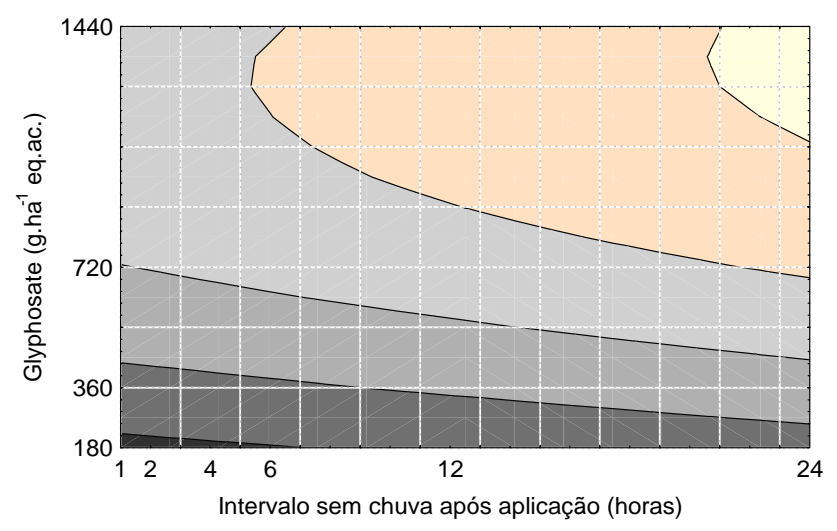

c)

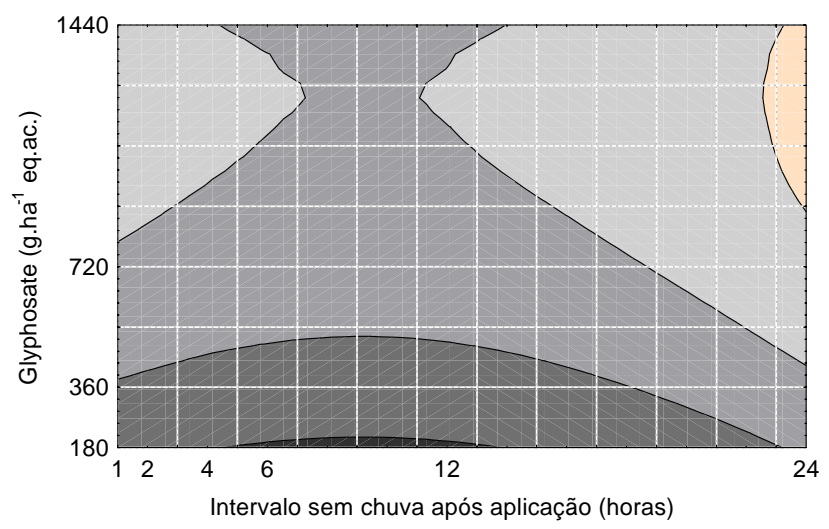

Figura 3 - Porcentagem de redução da matéria seca de Bidens pilosa aos 28 DAA, obtida pela relação entre a matéria seca dos tratamentos e a testemunha sem herbicida, em função das doses de Zapp Qi (sal potássico) (a), Roundup Transorb (sal isopropilamina) (b), Roundup WG (sal amônio) (c) e dos intervalos sem chuva após aplicação (20 mm em 30 minutos). 